\title{
Tratamento cirúrgico dos aneurismas toracoabdominais da aorta
}

Januário M. SOUZA*, Marcos F. BERLINCK*, Salomon O. ROJAS*, Dante F. SENRA*, Paulo A. F. OLIVEIRA* $^{*}$, José Renato M. MARTINS*, Ricardo MAZZIERI*, Sérgio Almeida de OLIVEIRA*

RBCCV 44205-127

SOUZA, J. M.; BERLINCK, M. F.; ROJAS, S. O.; SENRA, D. F.; OLIVEIRA, P. A. F.; MARTINS, J. R. M.; MAZZIERI, R.; OLIVEIRA, S. A. - Tratamento cirúrgico dos aneurismas toracoabdominais da aorta. Rev. Bras. Cir. Cardiovasc., 6(1): 17-23, 1991.

RESUMO: Foram operados, em nosso Serviço, 161 aneurismas da aorta, sendo 99 por dissecção e 62 por outras causas. Em cinco pacientes, os aneurismas eram de localizaçāo toracoabdominal, sendo três por degeneraçăo aterosclerótica e dois por dissecção; três pacientes eram do sexo feminino e a idade variou de 31 a 71 anos. Dois pacientes submeteram-se a aneurismectomia previamente (um da aorta ascendente e outro da porção proximal da aorta torácica). Revascularização miocárdica foi feita em um paciente, 40 dias antes da aneurismectomia. A indicação em todos os pacientes foi dor, causada por compressão do aneurisma, sendo que, em dois, havia insuficiência respiratória associada. Todos os pacientes foram operados através de incisão toracoabdominal e abertura do diafragma. A aorta foi substituída por tubo de Dacron, desde sua porção proximal até sua bifurcação, e as artérias viscerais foram implantadas no tubo. Quatro pacientes foram operados com pinçamento da aorta; um paciente necessitou emprego de circulação extracorpórea e parada circulatória, por impossibilidade de pinçamento da aorta junto à artéria subclávia. Todos os pacientes sobreviveram ao ato cirúrgico, ocorrendo dois óbitos no pós-operatório, um subitamente no $12^{\circ}$. dia e outro por coma neurológico secundário a parada cardiaca causada por hipóxia.

DESCRITORES: aneurismas toracoabodominais, cirurgia.

\section{INTRODUÇÃO}

A ressecção dos aneurismas da aorta de localização toracoabdominal apresenta muitos problemas: dificuldades no controle hemodinâmico do paciente, pelo pinçamento da aorta logo após a emergência da artéria subclávia; grande perda de sangue após abertura do aneurisma, pelas artérias viscerais, intercostais e lombares, exigindo reposição sangüinea rápida; dificuldade exposição; problemas técnicos para reconstrução da aorta $\Theta$ das artérias viscerais; possibilidade de isquemia da medula espinhal com paraplegia.

Em 1954, ENTHEREDGE et alii ${ }^{14}$ relatam ressecção com sucesso de um aneurisma da aorta abdominal alta envolvendo o tronco celiaco e a artéria mesentérica superior e reconstituição com enxerto homólogo e reimplante das artérias viscerais, usando uma incisão toracoabdominal.

DeBAKEY et alii ${ }^{10}$ utilizavam o enxerto homólogo de aorta no início da experiência. Posteriormente, introduziram o uso de prótese de Dacron para reconstrução da aorta e reimplante das artérias viscerais diretamente no tubo ou através de tubos menores também de Dacron, tendo publicado, em $1965^{\circ}$, uma série de 42 pacientes, com mortalidade de $26 \%$.

Em 1974, CRAWFORD ${ }^{3}$ descreveu técnica em que a exposição do aneurisma era inteiramente retroperitoneal. Não utilizava shunt temporário, mas simplesmente pinçamento da aorta acima e abaixo do aneurisma,

Trabalho realizado no Serviço do Prof. Dr. Sérgio Almeida de Oliveira. Hospital da Beneficência Portuguesa. Sāo Paulo, SP, Brasil.

Apresentado ao 18: Congresso Nacional de Cirurgia Cardiaca. Rio de Janeiro, RJ, 5 e 6 de abril, 1991.

* Do Serviço do Prof. Dr. Sérgio Almeida de Oliveira.

Endereço para separatas: Januário Souza. Rua Pará, 49, 3․ andar. 01243 São Paulo, SP. Brasil. 
que era aberto, substituindo-se a aorta por tubo de Dacron. As artérias viscerais (tronco celíaco, mesentérica superior e renais) eram implantadas diretamente no tubo, separadamente ou, quando possível, em bloco, diminuindo, assim. o tempo de isquemia visceral. Artérias intercostais e/ou lombares eram também reimplantadas, evitando-se isquemia medular. Em 1980, CRAWFORD \& SCHUESSLER ${ }^{6}$ publicaram uma série de 138 pacientes operados, com mortalidade global de $9 \%$. Excetuando-se as experiências de CRAWFORD el alii ${ }^{3,6,7}$ e de DeBAKEY et alii ${ }^{9,10}$, a literatura tem relatos de experiências com poucos casos, ou mesmo de casos isola$\operatorname{dos}^{12,13}, 15,18,20,23,24$. Esse fato motivou a apresentação de nossa experiência com o tratamento cirúrgico dessa doença.

\section{CASUISTICA E MÉTODOS}

Em nosso Serviço, foram operados 161 pacientes portadores de aneurisma da aorta, sendo que, em cinco deles, a localização era toracoabdominal. Desses cinco casos, três eram por degeneração aterosclerótica e dois por dissecção (Figuras 1 e 2). Três pacientes eram do sexo feminino. A idade variou de 31 a 71 anos, com média de 53 anos.

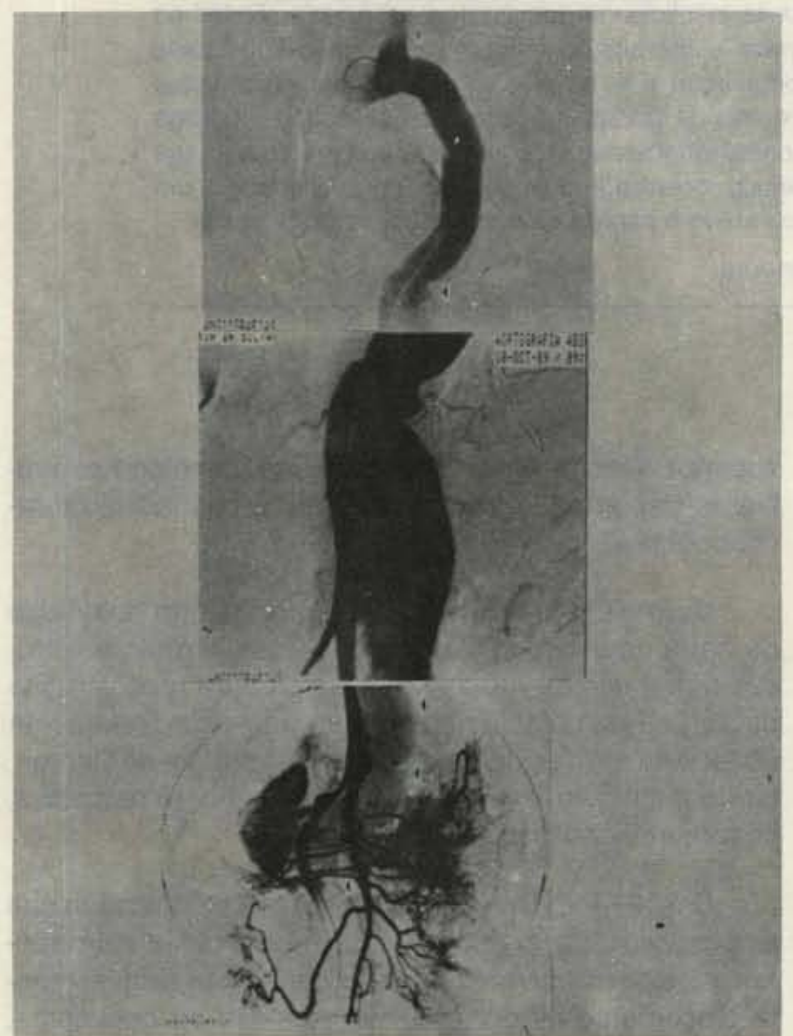

Fig. 1 - Aortografia pré-operatória de paciente submetido a correção de aneurisma dissecante toracoabdominal. Vendo-se tubo de Dacron da operaçăo prévia (aneurisma da aorta torácica), a dilataçāo aneurismática da luz falsa e a luz verdadeira comprimida, de onde emergem as artérias viscerais.

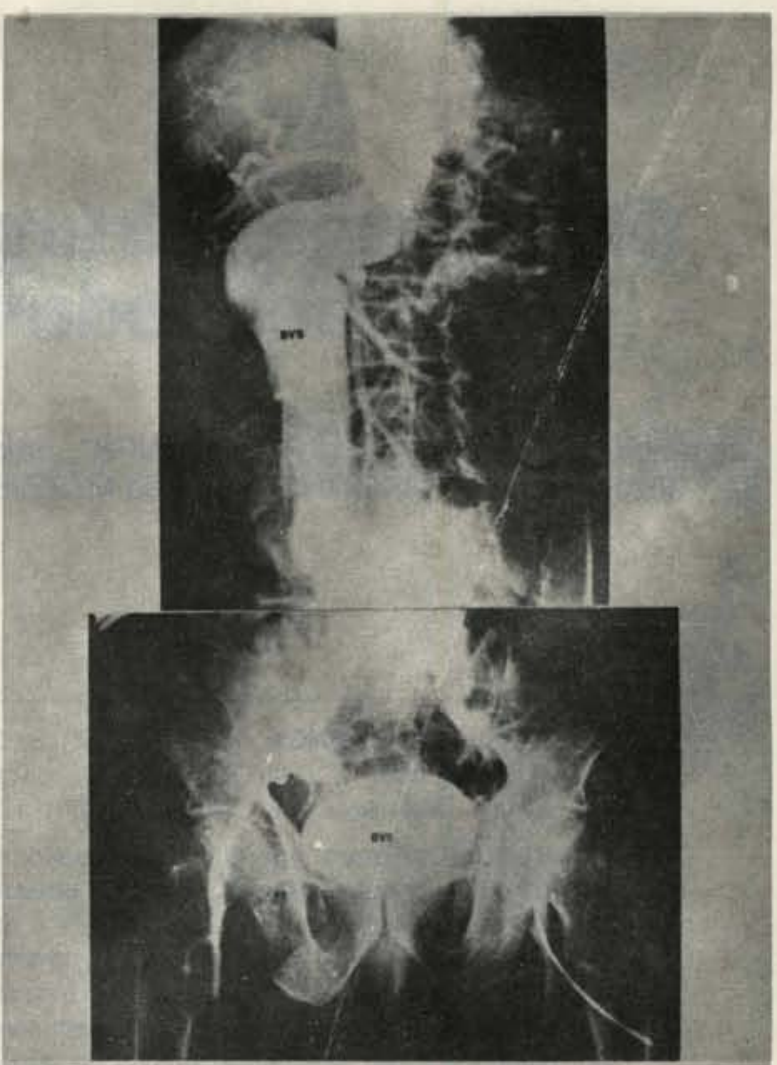

Fig. 2-Aortografia de paciente com aneurisma toracoabdominal por degeneração aterosclerótica envolvendo a aorta desde a origem da artéria subclávia esquerda e estendendo-se a ambas as artérias iliacas.

TABELA 1

\begin{tabular}{ll}
\hline \multicolumn{1}{c}{ Doenças Associadas } & N: Pacientes \\
\hline Hipertensão arterial sistólica & 5 \\
Insuficiência coronária & 2 \\
Paquipleurite & 1 \\
\hline
\end{tabular}

TABELA 2

\begin{tabular}{lc}
\hline \multicolumn{1}{c}{ Operaçōes prévias } & N: Pacientes \\
\hline Aneurisma aorta ascendente & 1 \\
Aneurisma aorta torácica & 1 \\
Revascularização miocárdica & 1 \\
\hline & TABELA 3 \\
\hline \multicolumn{1}{c}{ Manifestaçōes } & \\
\hline Dor torácica e abdominal & N: Pacientes \\
Massa pulsátil abdominal & 5 \\
Dispnéia & 5 \\
\hline
\end{tabular}


SOUZA, J. M.; BERLINCK, M. F.; ROJAS, S. O.; SENRA, D. F.; OLIVEIRA, P. A. F.; MARTINS, J. R. M.; MAZZIERI, R.; OLIVEIRA, S. A. - Tratamento cirúrgico dos aneurismas toracoabdominais da aorta. Rev. Bras. Cir. Cardiovasc., 6(1): $17-23,1991$.

Todos os pacientes tinham doenças associadas: hipertensão arterial sistêmica em cinco, insuficiência coronária em dois e paquipleurite em um (Tabela 1).

Três pacientes tiveram operações prévias, sendo ressecção de aneurismas da aorta em dois e revascularização miocárdica em outro paciente (Tabela 2 ).

Todos os pacientes eram sintomáticos, com dor e massa abdominal pulsátil em cinco e dificuldade respiratória por compressão do aneurisma em dois (Tabela 3).

Em três pacientes, o aneurisma estendia-se desde a origem da artéria subclávia esquerda até a bifurcação aorto-iliaca. Em um paciente, havia uma prótese de Dacron na porção torácica da aorta. Em outro paciente, o aneurisma estendia-se a ambas as artérias iliacas.

O diagnóstico foi suspeitado pelos sinais de sinto$\mathrm{m}$ s e pelos achados na radiografia simples de tórax. Enı ưês pacientes, foi utilizada cineangiografia e, em dois, foi utilizada angiografia digital. Três pacientes submeteram-se, também, a cineangiocoronariografia, sendo obsevadas lesões obstrutivas coronárias em dois.

A técnica utilizada na correção cirúrgica obedeceu, em linhas gerais, à sistematizaçāo descrita por CRAWFORD et alii ${ }^{7}$. A via de acesso foi através de incisão toracoabdominal. Em quatro pacientes, foi ressecada a quinta costela, possibilitando, assim, acesso à aorta junto à emergência da artéria subclávia. A toracotomia foi realizada no sétimo espaço intercostal no paciente previamente operado com substituição da aorta torácica por tubo de Dacron. O diafragma foi aberto em direção ao aneurisma. O abdome foi aberto por incisão xifopubiana. O peritônio foi incisado na goteira parieto-cólica esquerda, afastando-se para a direita o cólon descendente, baço, rim esquerdo, cauda e corpo do pâncreas, expondo-se, dessa maneira, a face látero-posterior do aneurisma.

Dado o colapso do pulmão esquerdo, foi indispensável o uso de sonda traqueal de dupla luz (Carlens).

A monitorização hemodinâmica deve ser a mais completa possível: cateter na artéria radial direita, para controle contínuo da pressão arterial; introdução de cateter de Swan-Ganz pela jugular interna direita, para monitorização da pressão venosa central e pressōes arterial e capilar pulmonar, já que ocorre grande alteração hemodinâmica pelo pinçamento da aorta torácica e pelo sangramento após abertura do aneurisma.

Em três pacientes, o clampeamento proximal da aorta foi feito logo após a subclávia esquerda. Em um paciente, foi pinçada a parte distal do tubo de Dacron previamente implantado. O pinçamentc di: al da aorta foi junto à sua bifurcação em três pacientes e em um paciente foram pinçadas as artérias iliacas comuns. O aneurisma foi, então, aberto em sua face látero-posterior, e um tubo de Dacron "women" de baixa porosidade pré coagulado com sangue autógeno, de $26 \mathrm{~mm}$ de diâmetro, foi anastomosado à porção proximal da aorta; as artérias mesentérica superior, tronco celíaco e renal direita foram implantadas em um único orifício do tubo, sendo a artéria renal esquerda implantada em orifício separado; pelo menos um par de artérias intercostais junto ao diafragma foi implantado ao tubo.

Em um paciente, por impossibilidade de pinçamento da aorta torácica proximal, foi utilizada a circulação extracorpórea entre átrio esquerdo e artéria femoral esquerda com hipotermia profunda $\left(15^{\circ} \mathrm{C}\right.$, esofágica) e parada circulatória de 47 minutos, reconstituindo-se a aorta com tubo de Dacron e implantando-se as artérias viscerais da mesma maneira.

Em um paciente, como havia envolvimento das artérias iliacas, foi usada distalmente uma prótese bifurcada de Dacron.

Um dos pacientes era portador de paquipleurite, o que dificultou a exposição do aneurisma e aumentou o sangramento.

O tubo de Dacron foi recoberto pela parede do aneurisma em quatro pacientes, sendo usado retalho de pericárdio bovino no quinto paciente, já que a parede do aneurisma era extremamente friável.

O paciente em que foi usada circulação extracorpórea apresentou sangramento intrapulmonar abundante com hipoxemia e parada cardiaca que, embora corrigidos, deixaram seqüela neurológica.

Nos quatro pacientes operados sem auxilio de circulação extracorpórea, não foi utilizada heparina.

Após implantação das artérias intercostais e viscerais, a pinça era deslocada distalmente, diminuindo-se, assim, o tempo de isquemia visceral.

\section{RESULTADOS}

Não houve óbito intra-operatório. Ocorreram dois óbitos no período de pós-operatório imediato. Um paciente faleceu no terceiro dia de pós-operatório, em coma neurológico conseqüente a hipoxemia e parada cardíaca ocorridos após o término da circulação extracorpórea, devido a sangramento intrapulmonar de difícil controle. O outro óbito ocorreu no 12: dia de pós-operatório, de maneira súbita, em paciente com quadro de insuficiência respiratória. O paciente já apresentava insuficiência respiratória antes da operação, por compressão pulmonar pelo aneurisma e por paquipleurite e havia sido submetido a revascularização miocárdica 40 dias antes.

Paraplegia em um paciente, que havia sido previamente operado para ressecção de aneurisma dissecante da aorta torácica. Duas artérias intercostais junto ao diafragma foram, como nos demais pacientes, implantadas no tubo de Dacron. 
Nenhum paciente apresentou insuficiência renal pós-operatória.

Foram mantidos jejum e sonda nasogástrica por cinco dias, sendo que nenhum paciente apresentou complicação gastrintestinal. Nesse período de jejum, foi estabelecida nutrição parenteral.

Em um paciente, o exame com ultra-som mostrou coleçāo líqüida retroperitonial, drenada através de pequena lombotomia no 10 : dia de pós-operatório, sendo evacuado aproximadamente $200 \mathrm{ml}$ de secreçāo sero-hemorrágica.

Transfusões de sangue homólogo foram necessárias em todos os pacientes, variando de quatro a sete bolsas por paciente.

O tempo de seguimento pós-operatório dos três sobreviventes foi, respectivamente, 13, 15 e 72 meses. Um paciente tem a limitaçāo da paraplegia. Os outros dois estāo assintomáticos, sendo que a paciente operada há 72 meses engravidou e teve uma gestaçāo com sucesso.

\section{DISCUSSĀO}

O tratamento cirúrgico dos aneurismas toracoabdominais da aorta tem apresentado ainda mortalidade elevada $(30 \%-50 \%)^{17,}{ }^{19}$. CRAWFORD et alii ${ }^{6,7}$ têm reportado resultados melhores, com mortalidade de $10 \%$, incluindo, aqui, os vários tipos de sua classificação.

A ocorrência de paraplegia e paraparesia, rara na correção de aneurismas da aorta ascendente, do arco aórtico e da aorta descendente é freqüênte na correção dos aneurismas de localização toracoabdominal; CRAWFORD \& SCHUESSLER ${ }^{6}$ relataram uma incidência de $12 \%$ em uma série de 138 pacientes, mais essa incidência chega a $21 \%$ nos 47 pacientes que tinham aneurisma tipo III, como os por nós aqui relatados. Não há uma relação bem estabelecida entre os métodos de proteçāo medular e a ocorrência de paraplegia ${ }^{1}, 4,5,8,11$. Em nossa experiência, ocorreu um caso de paraplegia em paciente previamente operado para correçāo de aneurisma da aorta descendente; técnica cirúrgica foi a mesma dos outros casos, sem qualquer acidente ou hemorragia, e duas artérias intercostais foram implantadas no tubo de Dacron, junto ao diafragma.
Não tivemos insuficiência renal no pós-operatớrio desses pacientes, o que está relatado na literatura ${ }^{17}$, numa porcentagem que varia de $3 \%$ a $15 \%$, inclusive com casos de irreversibilidade, e os pacientes em programa de hemodiálise. As causas mais freqüentes săo a idade avançada e a hipertensão arterial. A maioria dos autores năo usa procedimento especial de proteçăo renal, havendo os que utilizam perfusão renal com soro gelado ${ }^{16}$.

Também tivemos hemorragia seja no intra ou pósoperatório imediato.

A complicação grave que ocorreu no intra-operattório foi insuficiência respiratória aguda causada por sangra. mento intrapulmonar em paciente que, por dificuldades técnicas, foi necessária a utilizaçăo de circulação extracorpórea.

O uso de circulação extracorpórea com hipotermia e parada circulatória é indispensável em casos de dificuldades técnicas e em casos de acidente; há, no entanto, maior incidência de complicaçōes pulmonares ${ }^{4}$, dévido a traumatismo pulmonar por retraçāo, que é mal tolerada nos pacientes heparinizados.

Em outro paciente, a insuficiência respiratória, já presente no pré-operatório, indicou a realização de traqueostomia e assistência ventilatória prolongada, o que é comumente relatado na literatura.

A sobrevida a longo prazo (cinco anos) relatada na literatura é de $62 \%$ a $64 \%{ }^{7}, 17$. Nossos três pacientes que sobreviveram estăo em bom estado geral, excetuando-se o paciente que tem limitação pela paraplegia.

A técnica descrita por BORST et alii ${ }^{2}$ tem grande utilidade quando existe aneurisma toracoabdominal associado a aneurisma da aorta ascendente e/ou do arco aórtico, sendo esses corrigidos em primeiro lugar, por esternotomia mediana, deixando um tubo flutuante na aorta descendente, corrigindo-se posteriormente $o$ aneurisma toracoabdominal.

O melhor conhecimento das causas da paraplegia pós-operatória e, conseqüentemente, sua prevençăo é, atualmente, um dos pontos de maior interesse para aqueles que estão envolvidos com esse tipo de operaçăo $4,5,8,11$. 
SOUZA, J. M.; BERLINCK, M. F.; ROJAS, S. O.; SENRA, D. F.; OLIVEIRA, P. A. F.; MARTINS, J. R. M.; MAZZIERI, R.; OLIVEIRA, S. A. - Surgical treatment of thoracoabdominal aortic aneurysms. Rev. Bras. Cir. Cardiovasc., 6(1): 17-23, 1991.

ABSTRACT: Five patients have been operated on of thoracoabdominal aortic aneurysms. The mean age was 53 years (range $31-71$ ) and three were women. All the patients were symptomatic, three of them had arterioscierotic aneurysms, and the other two had dissecting aneurysms. Three patients had been operated on previously. The exposure of aneurysm was made through a thoracoabdominal incision, in four patients clamps were placed above and below the aneurysm and it was incised longitudinally. Bypass between left atrium and left femoral artery with hypothermia and circulatory arrest was used in the other patients, since the proximal thoracic aortic clamping was impossible. A woven Dacron graft of adequate size was used to substitute the aorta, intercostals and visceral arteries were reimplanted. Paraplegia occurred in one patient. There was no intraoperative death. Two patients died in the immediate postoperative period, one of them on the 3rd day, by neurologic coma caused by cardiac arrest during the operation, and the other patient on the 12th postoperative day, suddenlly. Three patients were followed up. Two patients are doing well, 13 and 72 months after operation, and the other one has the limitation of the paraplegia.

DESCRIPTORS: aneurysms, thoracoabdominal, surgery.

\section{REFERÊNCIAS BIBLIOGRÁFICAS}

1 ADAMS, H. D. \& GEERTRYDEN, H. H. - Neurologic compplications of aortic surgery. Ann. Surg., 144: 574-610, 1956.

2 BORST, H. G.; WALTERBUSCH, G.; SCHAPS, D. - Extensive aortic replacement using "elephant trunk" prosthesis. Thorac. Cardiovasc. Surgeon, 31: 37-40, 1983.

3 CRAWFORD, E. S. - Thoracoabdominal and abdominal aortic aneurysms involving renal, superior mesenteric and celiac arteries. Ann. Surg., 179: 763-772, 1974.

4 CRAWFORD, E. S.; COSELLI, J. S.; SAFI, H. J. - Partial cardipulmonary bypass, hypothermic circulatory arrest and posterolateral exposure for thoracic aortic aneurysm operation. J. Thorac. Cardiovasc. Surg., 94: 824-827, 1987.

5 CRAWFORD, E. S.; MIZRAHI, E. M.; HESS, K. P.; COSELLI, J. S.; SAFI, H. J.; PATEL, V. M. - The impact of distal aortic perfusion and somatosensory evoked potential monitoring on preservation of paraplegia after aortic aneurysms operation. J. Thorac. Cardiovasc. Surg., 95: 357-367, 1988.

6 CRAWFORD, E. S. \& SCHUESSLER, J. S. - Thoracoabdominal and abdominal aortic aneurysms involving celiac, superior mesenteric and renal arteries. World J. Surg., 4: 643-652, 1980.

7 CRAWFORD, E. S.; SNEYDER, D. M.; CHO, G. C.: ROEHM, J. O. F. - Progress in treatment of thoracoabdominal and abdominal aortic aneurysms involving celiac, superior mesenteric and renal arteries. Ann. Surg., 188: 404-422, 1978.

8 CUNNINGHAM, J. N.; LASCHINGER, J. C.; MERKIN, H. A.; NATHAN, I. M.; COLVIN, S.; RAUSOHUFF, J.; SPENCER, F. C. - Measurement of spinal cord ische- mia during operations upon the thoracid aorta. Ann. Surg., 196: 285-296, 1982.

9 DeBAKEY, M. E.; CRAWFORD, E. S.; GARRET, H. E.; BEALL, A. C.; HOWELL, J. F. - Surgical considerations in the treatment of aneurysms of thoracoabdominal aorta. Ann. Surg., 162: 650-662, 1965.

10 DeBAKEY, M. E.; CREECH, O.; MORRIS, G. C. - Aneurysm of thoracoabdominal aorta involving the celiac, superior mesenteric and renal arteries: report of four cases treated by ressection and homograft replacement. Ann. Surg., 144: 549-573, 1956.

11 DRUMMOND, J. C.; ZORNOW, M. H.; DEMBITSKY, W. P. - Spurious identification of critical intercostal arteries by evoked responses during aortic resection. Ann. Thorac. Surg., 44: 79-81, 1987.

12 EDMUNDS, L. H. \& FOKMAN, M. J. - Aneurysmoplasty and prosthetic bypass for aneurysms of the descending thoracic and thoracoabdominal aorta. J. Thorac. Cardiovasc. Surg., 52: 395-407, 1967.

13 EDWARDS, W. S. - Thoracoabdominal aortic aneurysms. Surg. Clin. N. Am., 62: 441-448, 1982.

14 ENTHEREDGE, S. N.; YEE, J.; SMITH, J. V.; SCHONBERGER, S.; GOLDMAN, M. J. - Successful resection of a large aneurysm of the upper abdominal aorta and replacement with homograft. Surgery, 38: 1071-1081, 1955.

15 HARDY, J. M.; TIMMIS, H. H.; SALEH, S. J.; HARDIN, W. S. - Thoracoabdominal aortic aneurysm: simplified surgical management with case report. Ann. Surg., 166: 1008-1011, 1967.

16 HUMMEL, B. W. \& ROSSI, N. P. - A simple technique for renal protection during repair of complicated aneurysms. Ann. Thorac. Surg., 39: 584-585, 1985. 
SOUZA, J. M.; BERLINCK, M. F.; ROJAS, S. O.; SENRA, D. F.; OLIVEIRA, P. A. F.; MARTINS, J. R. M.; MAZZIERI, R.; OLIVEIRA, S. A. - Tratamento cirúrgico dos aneurismas toracoabdominais da aorta. Rev. Bras. Cir. Cardiovasc., 6(1): 17-23, 1991.

17 KIRKLIN, J. W. \& BARRAT-BOYES, B. G. - Cardiac surgery. New York, John Wiley \& Sons, 1986. p. 1493-1524.

18 KOROMPAI, F. L. \& HAYWARD, R. H. - Preservation of visceral perfusion during resection of thoracoabdominal aneurysm. Bull. Texas Heart Inst., 2: 349-351, 1975.

19 MASSIMO, C. G.; POMA, A. G.; VILIGIARDI, R. R.; DURANTI, A.; COLUCCI, M.; FAVI, P. P. - Simultaneous total aortic replacement from arch to bifurcation experience with six cases. Texas Heart Inst. J., 13: $147-151,1986$.

20 PAPADOPUOLOS, C. D.; MANOLI, A. M.; SOBEL, S. Surgical treatment of thoracoabdominal aneurysms. J. Cardiovasc. Surg., 12: 456-462, 1971.

21 POKELA, R.; KÄRKÖLÄ, P.; TARKKA, M.; KAIRAMONA, M. I.; LARMI, T. K. I. - Surgery of thoracoabdominal aortic aneurysms. Scand. J. Thorac. Cardiovasc. Surg., 18: 179-189, 1984.

22 SCHUMAKER, H. B. - Innovation in the operative management of the thoracoabdominal aortic aneurysm. Surg. Gynecol. Obstet., 136: 793-794, 1973.

23 SELLE, J. G.; ROBICSECK, F.; DAUGHERTY, H. K.; COOK, J. W. - Thoracoabdominal aortic aneurysms: a review and current status. Ann. Surg., 189: 158-164, 1979.

24 STONEY, R. J. \& WYLIE, E. J. - Surgical management of arterial lesions of the thoracoabdominal aorta. Am. J. Surg., 126: 157-164, 1973.

AGRADECIMENTO: Agradecemos ao Dr. Júlio César Saucedo Mariño, pela sua participação na operação de um dos pacientes.

\section{Discussão \\ DR. ÊNIO BUFFOLO \\ São Paulo, SP}

Gostariamos, inicialmente, de cumprimentar o $\mathrm{Dr}$. Januário, pela oportunidade de trazer à discussāo uma doença da maior gravidade que, para sua correção, exige equipe altamente diferenciada, habilidade e, acima de tudo, coragem para enfrentar um risco operatório muito alto. Nossa experiência cirúrgica é essencialmente parecida com a apresentada pelos autores e, de um total de 235 lesões operadas da aorta torácica, tivemos apenas quatro casos de aneurismas toracoabdominais. Ressaltaríamos alguns aspectos que, a nosso ver, são de impotância: 1) Quanto à abordagem, utilizamos a toracotomia esquerda, com o paciente entubado seletivamente, excluindo-se o pulmão esquerdo. Nos casos em que existe coto após a subclávia esquerda, não utilizamos circuito extracorpóreo, mas, naquelas situações nas quais o aneurisma é muito volumoso prejudicando a dissecção proximal, ou, ainda, naqueles casos nos quais não existe coto proximal definido, preferimos realizar a derivação extracorpórea parcial veia-artéria femoral com interposição de oxigenador, levando o paciente a hipotermia profunda $20^{\circ} \mathrm{C}$ e parada circulatória. Nessas condiçōes, o aneurisma é incisado e fica muito fácil a sutura proximal. Terminado esse tempo, que é, habitualmente, rápido, restabelecemos a circulação extracorpórea, agora com a cânula arterial posicionada na aorta ascendente, e pinçamos o tubo de Dacron, prosseguindo a operação. A desvantagem da necessidade de heparinização é compensada pela possibilidade de aspiração de reaproveitamento do sangue. 2) No que diz respeito ao risco da paraplegia, complicação temíveie desastrosa de incidência maior na correção deste tipo de lesão, não conhecemos, da literatura compulsada, maneira eficiente de preveni-la. A aferição dos potenciais evocados durante o pinçamento aórtico não mostrou correlação confiável. Crawford, que detém a maior e melhor experiência mundial neste campo, aconselha o reimplante do tubo de dois pares de intercostais, escolhendo-se os pares mais calibrosos. 3) Acreditamos que a dificuldade maior encontrada neste tipo de intervenção diz respeito à abordagem do coto proximal. A escolha do nível da toracotomia, se alta, favorece esse acesso, mas, em compensação, dificulta o manuseio na aorta torácica baixa. Uma manobra útil é a abordagem através de dois intercostos: o 4: ou 5: para o coto proximal e outra incisão intercostal baixa 7 : intercosto para a porção distal, prosseguindo-se a operação como os autores preconizam. Gostariamos, finalmente, de perguntar ao Dr. Januário se o paciente que havia sido revascularizado 40 dias antes era portador de aneurisma dissecante, se, em seus cinco casos, preocupou-se em reimplantar a mesentérica inferior, sendo este aspecto polêmico na literatura, e, finalmente, se ocorreu e como manejaram a hipertensāo arterial proximal durante o pinçamento da aorta após a subclávia. Reinteramos, ao final deste comentário, a excelência da apresentação deste assunto, ressaltando que em 18 Congressos de nossa Sociedade, é a primeira vez que este assunto é abordado de maneira corajosa e com bons resultados.

\section{DR. HENRIQUE MURAD \\ Rio de Janeiro, RJ}

Agradecemos à Comissão Organizadora do 18 : Congresso Nacional de Cirurgia Cardiaca a oportunidade de comentar este trabalho. Parabenizamos os autores, pelo modo como conduziram estes difíceis casos. No Serviço de Cirurgia Cardiaca e Serviço de Cirurgia Vascular do Hospital Universitário Clementino Fraga Filho, da UFRJ, foram realizadas cirurgias para tratamento de aneurismas toracoabdominais em 12 pacientes. Gostaríamos de tecer algumas consideraçōes sobre as técnicas usadas nessas operaçōes. Em nove pacientes, foi usada a técnica clássica preconizada por Crawford. Qua- 
SOUZA, J. M.; BERLINCK, M. F.; ROJAS, S. O.; SENRA, D. F.; OLIVEIRA, P. A. F.; MARTINS, J. R. M.; MAZZIERI, R.; OLIVEIRA, S. A. - Tratamento cirúrgico dos aneurismas toracoabdominais da aorta. Rev. Bras. Cir. Cardiovasc., 6(1): 17-23, 1991.

tro pacientes tiveram excelente evoluçāo. Quatro faleceram como conseqüência de complicaçōes cirúrgicas (hemorragia pulmonar, insuficiência respiratória e discrasia sangüínea) e um paciente permanece paraparético. Em dois pacientes, usamos derivações para os vasos viscerais a partir da aorta descendente, durante o pinçamento aórtico. Essa técnica foi descrita por Korompai e colaboradores, em 1975. (Bull. Texas Heart. Inst., 2: 349,1975$).$ Cânulas de perfusão coronariana tipo De Bakey foram usadas para nutrir os vasos viscerais. Em um dos pacientes, houve, inclusive, produção de urina durante o pinçamento aórtico. Esses dois pacientes estão vivos, sendo que um deles permanece bem, 12 anos após a cirurgia. Um último paciente, operado há quatro anos para ressecção de aneurisma de aorta ascendente e troca de valva aórtica, foi submetido com sucesso a ressecção de aneurisma toracoabdominal com parada circulatória total. Neste caso, usamos a técnica de CEC, preconizada por Kouchouko (J. Thorac. Cardiovasc. Surg., 99: 659, 1990). O retorno venoso foi obtido por cânulas introduzidas pela veia femoral e artéria pulmonar, e o retorno arterial foi obtido pela artéria femoral. Ao fim da anastomose proximal, uma cânula colocada através de enxerto permitiu o reinício da perfusão cerebral e retirada de ar do arco aórtico. Em todos esses casos, foi usado acesso retroperitonial e retro-renal esquerdo à aorta abdominal. A pré-coagulação do enxerto foi feita com plasma homólogo ou albumina em autoclave a $120 \mathrm{~F}$ por cinco minutos. Os enxertos usados foram tipo "very soft woven" (Vaskutek, Meadox). Recentemente, começamos a usar enxertos de Dacron revestidos internamente por pericárdio bovino (Labcor), obtendo ausência total de extravazamento sangüíneo através do enxerto. Nos casos de dissecção aórtica, usamos cola biológica (ColagelCirumédica) e reforço da sutura com feltro de Teflon. Para evitar paraplegia, foram reimplantadas artérias intercostais sempre que possível, usado corticosteróides e drogas que neutralizam radicais livres (alopurinol, tocoferol). Não temos experiência com drenagem de líqüido céfalo-raquidiano ou a injeção de papaverina intrate- cal preconizados por Jversson (Ann. Surg., 204: 38, 1986).

\section{DR. SOUZA \\ (Encerrando)}

Agradeço os comentários do Dr. Murad e o cumprimento pelos seus resultados. A denominação toracoabdominal engloba aneurismas de extensão variável e a classificação proposta por Crawford é de grande utilidade, para compararmos graus de dificuldade e de risco cirúrgico. Realmente, os enxertos de Dacron com revestimento interno de pericárdio são um grande avanço; infelizmente, esses enxertos ainda sāo fornecidos com apenas $20 \mathrm{~cm}$ de comprimento: portanto, não podem ser utilizados em aneurismas de grande extensāo. Ao Professor Buffolo, agradeço os estimulantes comentários e concordo em que a circulação extracorpórea com hipotermia e parada circulatória facilita a anastomose proximal do tubo à aorta. Em nossos pacientes, reimplantamos no tubo duas ou três artérias intercostais da porção terminal da aorta torácica, o que, segundo Crawford, diminui a incidência de paraplegia. O paciente previamene operado para revascularização miocárdica era potador de extenso aneurisma aterosclerótico. Em nenhum dos pacientes a artéria mesentérica inferior foi reimplantada, pois estava acluída. Finalmente, quanto à hipertensāo arterial proximal, que sempre ocorre após o pinçamento, foi controlada com infusōes de nitroprussiato de sódio - um recurso que pode também ser usado é a utilização de bomba centrífuga com bypass átrio esquerdo-femoral, mas que não utilizamos. Agradeço os comentários do Prof. Domingos Junqueira de Morais, e, quanto ao seu trabalho publicado na revista "O Hospital", teria imenso prazer em citá-lo; portanto, gostaria de receber uma separata. Dr. Paulo Paulista, muito obrigado por seus comentários; quanto à sua pergunta sobre problemas com o baço, houve rotura em um paciente, sendo necessária a esplenectomia. 